\title{
Dioscin enhances osteoblastic cell differentiation and proliferation by inhibiting cell autophagy via the ASPP2/NF-кß pathway
}

\author{
CHEN ZHU, NIRONG BAO, SHUO CHEN and JIANNING ZHAO
}

\author{
Department of Orthopaedic Surgery, Jinling Hospital, \\ Nanjing University School of Medicine, Nanjing, Jiangsu 210002, P.R. China
}

Received March 3, 2016; Accepted February 13, 2017

DOI: $10.3892 / \mathrm{mmr} .2017 .7206$

\begin{abstract}
Dioscin, a typical steroid saponin, has been reported to promote osteoblastic cell differentiation. However, the underling mechanisms remain to be elucidated. In the present study, it was identified that dioscin $(0.5,1,5,10$ and $25 \mu \mathrm{g} / \mathrm{ml})$ promoted MC3T3-E1 cell proliferation and differentiation in a dose-dependent manner. Western blot analysis showed that dioscin regulated autophagy-associated protein expression in MC3T3-E1 cells; it promoted the expression of apoptosis stimulated protein of p53-2 (ASPP2), and inhibited the expression of nuclear factor (NF)- $\kappa \beta$ and microtubule-associated protein 1 light chain $3 \beta$, in a concentration-dependent manner. Caffeic acid phenethyl ester (CAPE) was used to inhibit the activation of NF- $\kappa \mathrm{B}$ and examine the effect of the ASPP $2 / \mathrm{NF}-\kappa \beta$ pathway on osteoblastic cell differentiation, proliferation and autophagy. It was identified that CAPE reversed the regulation of dioscin on osteoblastic cell differentiation, proliferation and autophagy. In conclusion, the present study revealed that dioscin promoted osteoblast proliferation and differentiation by inhibiting cell autophagy via the ASPP $2 / \mathrm{NF}-\kappa \beta$ pathway. These results are the first, to the best of our knowledge, to reveal the involvement of autophagy in the effects of dioscin on the prevention and therapy of osteoporosis.
\end{abstract}

\section{Introduction}

Dioscin, a typical steroid saponin extracted from Polygonatum zanlanscianense Pamp., was first isolated and characterized in the mid-20th century (1). Dioscin exhibits cytotoxicity against a number of human malignant cell lines and has been investigated extensively for the treatment of various types of cancer (2-5). Previous pharmacological studies have demonstrated that dioscin also has antispasmodic activity (6) antithrombotic activity (7), and protects against liver damage (8) and diabetes mellitus (9).

Correspondence to: Dr Jianning Zhao, Department of Orthopaedic Surgery, Jinling Hospital, Nanjing University School of Medicine, 305 East Zhongshan Road, Nanjing, Jiangsu 210002, P.R. China

E-mail: zhaojianning00207@163.com

Key words: dioscin, osteoblastic differentiation, autophagy, apoptosis stimulated protein of $\mathrm{p} 53-2$, nuclear factor- $\kappa \beta$
Associated studies have demonstrated that Dioscorea plants have an effect in preventing and treating osteoblastic differentiation and proliferation. Zhang et al demonstrated that dioscin promotes osteoblastic proliferation and differentiation via low-density lipoprotein receptor-related protein 5 and the endoplasmic reticulum pathway (10). It is well known that autophagy, a conserved lysosomal degradation pathway, is closely associated with cell differentiation and development. Ha et al showed that autophagy was involved in bioactive silica nanoparticle-induced osteoblast differentiation (11). However, whether autophagy is involved in dioscin-induced osteoblastic proliferation remains to be elucidated.

Apoptosis stimulated protein of p53-2 (ASPP2), a pro-apoptotic regulator and member of the ASPP family, is a p53-binding protein, which specifically stimulates the pro-apoptotic function of p53 (12). Previous studies have demonstrated that the function of ASPP2 is more complex than simply enhancing p53-mediated apoptosis, and its binding partners are involved in various biological pathways (13). Liu et al reported that ASPP2 induces autophagic apoptosis by releasing Beclin 1 from cytoplasmic B-cell lymphoma 2-Beclin-1 complexes in hepatoma cells (14) and inhibits RAS-induced autophagy by preventing the formation of autophagy-related (ATG) 16/ATG5/ATG12 (15). ASPP2 has also been reported to protect against hepatocyte injury by reducing autophagy (16). The transcription factor, nuclear factor $(\mathrm{NF})-\kappa \beta$ is a protein complex, which controls the transcription of DNA, cytokine production and cell survival. A previous study demonstrated ASPP2 as an NF- $\kappa \beta$ inhibitor, and showed that ASPP2 binds to two sites in NF- $\kappa \beta$ (p65), at residues 236-253 and 293-313, which contain the nuclear localization signal (17). However, the role of the ASPP2/NF- $\mathrm{B}$ pathway in dioscin-induced osteoblastic proliferation remains to be elucidated.

In the present study, the mechanism by which dioscin prevents osteoporosis was investigated. It was identified that dioscin promoted osteoblast proliferation and differentiation by inhibiting cell autophagy via the ASPP $2 / \mathrm{NF}-\kappa \beta$ pathway, revealing the mechanism underlying the effect of dioscin on the prevention and therapy of osteoporosis.

\section{Materials and methods}

Cell culture. The MC3T3-E1 cells were obtained from American Type Culture Collection (Manassas, VA, USA) and 
cultured in DMEM (Hyclone, Logan, UT, USA) supplemented with $10 \%$ FBS (Hyclone), $100 \mathrm{U} / \mathrm{ml}$ penicillin and $100 \mathrm{mg} / \mathrm{ml}$ streptomycin. The cells were cultured at $37^{\circ} \mathrm{C}$ in a humidified atmosphere of $5 \% \mathrm{CO}_{2}$. Then, the cells $\left(\sim 2 \times 10^{5}\right)$ were treated with dioscin $(0.1,0.5,1,5,10$ and $25 \mu \mathrm{g} / \mathrm{ml}$; HY-N0124; Medchemexpress, Monmouth Junction, NJ, USA) for 24, 48 and 72 h. CAPE $(1 \mu \mathrm{M}$; S7414; Selleck Chemicals, Houston, TX, USA) was used to inhibit p-NF- $\mathrm{kB}-\mathrm{P} 65$.

Cell proliferation assay. Cell proliferation was measured using a 3-dimethylthiazol-2-y-(4,5)-2, 5-diphenyltetrazolium bromide (MTT) assay. The MC3T3-E1 cells were seeded in 96 -well culture plates at a density of $5 \times 10^{3}$ per well. The medium was removed and the cells were treated with dioscin $(0.1,0.5,1,5,10$ and $25 \mu \mathrm{g} / \mathrm{ml})$ for 24,48 and $72 \mathrm{~h}$. Subsequently, MTT (10 $\mu$ l per well; $5 \mathrm{mg} / \mathrm{ml}$ ) solution was added to each well and incubated at $37^{\circ} \mathrm{C}$ for $4 \mathrm{~h}$. Finally, the optical density was determined at $570 \mathrm{~nm}$ using an ELISA plate reader (Model 550; Bio-Rad Laboratories, Inc., Hercules, CA, USA).

Western blot analysis. Total proteins were extracted from the MC3T3-E1 cells in RIPA buffer with $0.5 \%$ sodium dodecyl sulfate (SDS) and 3\% proteinase inhibitor cocktail (Sigma-Aldrich; Merck KGaA, Darmstadt, Germany) for $30 \mathrm{~min}$ on ice. The concentration of protein was determined using a BCA protein assay kit (Santa Cruz Biotechnology, Inc., Dallas, TX, USA). For western blot analysis, the proteins were separated by $10 \%$ SDS-PAGE and transferred onto PVDF membranes (Invitrogen; Thermo Fisher Scientific, Inc., Waltham, MA, USA). Following blocking with 5\% bovine serum albumin (A-3912; Sigma-Aldrich; Merck KGaA) at room temperature for $1 \mathrm{~h}$, the membranes were incubated at $4^{\circ} \mathrm{C}$ overnight with the following polyclonal antibodies: ASPP2 (ab36004; 1:1,000; Abcam Biotechnology, Cambridge, UK), phosphorylated (p)-NF- $\kappa \beta$ p65 (sc-166748; 1:1,000; Santa Cruz, CA, USA), alkaline phosphatase (ALP; ab133602; 1:10,000; Abcam), microtubule-associated protein 1 light chain 3 $\beta$ (LC3B; 2775; 1:800; Cell Signaling Technology, Inc, Shanghai, China), bone gla protein (BGP; sc-74,495; 1:400; Santa Cruz) and GAPDH (sc-365062; 1:500; Santa Cruz). The membranes were washed in PBS 3 times for 10 mins and then incubated with horse radish peroxidase-conjugated secondary antibodies that against mouse IgG (A9309; Sigma-Aldrich; Merck KGaA) or rabbit IgG (ab97051; Abcam) with 1:2,000 dilution for $2 \mathrm{~h}$ at room temperature. The intensities of the bands were quantified using an image analysis system (Odyssey; LI-COR Biosciences, Lincoln, NE, USA).

Immunofluorescence assay. The cells were seeded on polylysine-coated glass slides (Sigma-Aldrich; Merck Millipore) in 6-well plates with a density of $1-5 \times 10^{5} / \mathrm{ml}$, fixed in $4 \%$ paraformaldehyde for $15 \mathrm{~min}$, washed with PBS and permeabilized with $0.1 \%$ Triton-X-100 (Sigma-Aldrich; Merck Millipore) for $5 \mathrm{~min}$. The slides were then incubated with LC3 primary antibody (L8918; 1:1,000; Sigma-Aldrich; Merck $\mathrm{KGaA}$ ) for 1 hatroom temperature and incubated with secondary antibody (fluorescence FITC; KC-RB-095; 1:100; Kangcheng Biotech, Shanghai, China) for $1 \mathrm{~h}$ at room temperature. DAPI (Invitrogen; Thermo Fisher Scientific, Inc.) was added to the cells in the dark for $5 \mathrm{~min}$. Following washing with
PBS, the glass slides were sealed with seal sheet containing fluorescence quenching agent. A laser-scanning confocal fluorescence microscope (Olympus BX60; Olympus, Tokyo, Japan) was used to capture images.

Statistical analysis. All experiments were repeated at least three times with similar results. Representative data are given. Statistical analysis was performed using paired Student's t-tests. $\mathrm{P}<0.05$ was considered to indicate a statistically significant difference. All data were analyzed with SPSS version 17.0 (SPSS Inc., Chicago, IL, USA).

\section{Results}

Effects of dioscin on MC3T3-E1 cell proliferation, differentiation and autophagy. To analyze the effects of dioscin on MC3T3-E1 cell proliferation, the MC3T3-E1 cells were incubated with dioscin at various concentrations $(0.1,0.5,1,5,10$ and $25 \mu \mathrm{g} / \mathrm{ml}$ ) and cell proliferation was detected using MTT assays. As demonstrated in Fig. 1A, the results showed that dioscin concentrations of $0.5,1,5,10$ and $25 \mu \mathrm{g} / \mathrm{ml}$ significantly promoted MC3T3-E1 cell proliferation in $72 \mathrm{~h}$ in a concentration-dependent manner, compared with the control cells.

ALP, a representative marker of the early stage of osteoblast differentiation, is known to be important in the initiation of mineralization during bone formation. Osteoblastic differentiation was characterized by the activity of ALP. LC 3B is a marker of autophagy. The present study analyzed the expression of ALP and LC3B in the MC3T3-E1 cells in response to dioscin. As demonstrated in Fig. 1B-D, dioscin concentrations of $0.5,1,5,10$ and $25 \mu \mathrm{g} / \mathrm{ml}$ promoted the expression of ALP and inhibited the expression of LC3B in MC3T3-E1 cells, in a concentration-dependent manner.

Effects of dioscin on the expression of ASPP 2 and NF- $\kappa \beta$ in MC3T3-E1 cells. ASPP2 has been reported to be associated with autophagy and to regulate the expression of NF- $\kappa \beta$. In the present study, the effects of dioscin on the expression of ASPP2 and NF- $\kappa \beta$ in MC3T3-E1 cells were detected. As demonstrated in Fig. 2, dioscin concentrations of 1, 5, 10 and $25 \mu \mathrm{g} / \mathrm{ml}$ decreased the expression of ASPP2 and promoted the expression of NF- $\kappa \beta$ in the MC3T3-E1 cells, in a concentration-dependent manner.

$N F-\kappa \beta$ is involved in dioscin-induced osteoblastic cell proliferation and differentiation. A previous study showed that ASPP2 can inhibit autophagy through the p53-independent pathway (12) and NF- $\kappa \beta$ was reported to be target protein of ASPP2, with two sites at residues 236-253 and 293-313 (17). To determine the effects of the ASPP $2 / \mathrm{NF}-\kappa \beta$ pathway in dioscin-induced osteoblastic cell differentiation and proliferation, CAPE was used to inhibit the activation of NF- $\mathrm{kB}$, and osteoblastic cell differentiation and proliferation were examined using western blot analysis and an MTT assay. As demonstrated in Fig. 3A, CAPE treatment inhibited the expression of $\mathrm{p}-\mathrm{NF}-\mathrm{\kappa} \beta$, but did not affect the expression of ASPP2. CAPE reversed dioscin-induced osteoblastic cell differentiation by repressing the expression of ALP and BGP. CAPE also attenuated dioscin-induced osteoblastic cell 


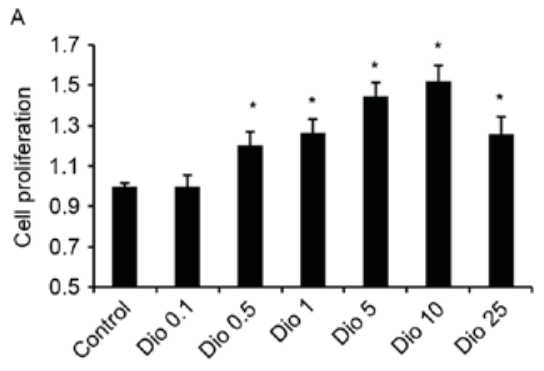

C

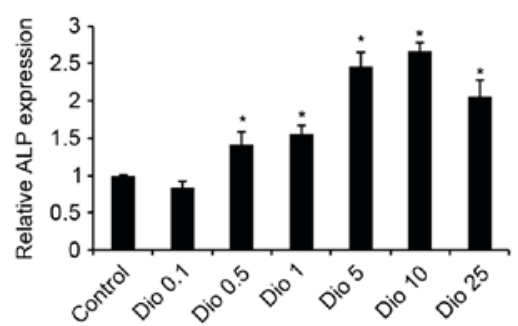

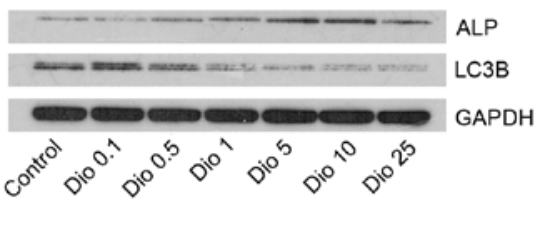

D

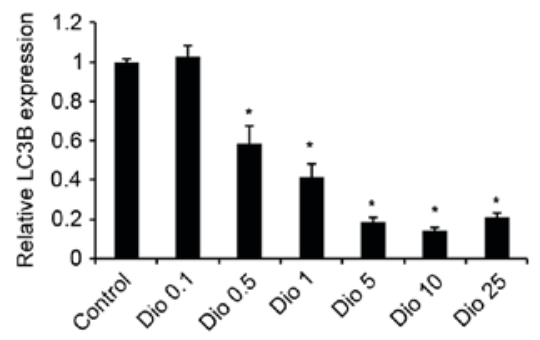

Figure 1. Effects of dioscin on MC3T3-E1 cell proliferation, differentiation and autophagy. (A) Effects of dioscin on proliferation in MC3T3-E1 cells. MC3T3-E1 cells were treated with dioscin $(0.1,0.5,1,5,10$ and $25 \mu \mathrm{g} / \mathrm{ml})$ for $72 \mathrm{~h}$. Cell proliferation was assessed using a 3-dimethylthiazol-2-y-(4,5)-2, 5-diphenyltetrazolium bromide assay. (B) Effects of dioscin on the expression of (C) ALP and (D) LC3B in MC3T3-E1 cells. Results were obtained from three independent experiments and are expressed as the mean \pm standard deviation. ${ }^{*} \mathrm{P}<0.01$, vs. control. Dio, dioscin; ALP, alkaline phosphatase; LC3B, microtubule-associated protein 1 light chain $3 \beta$.
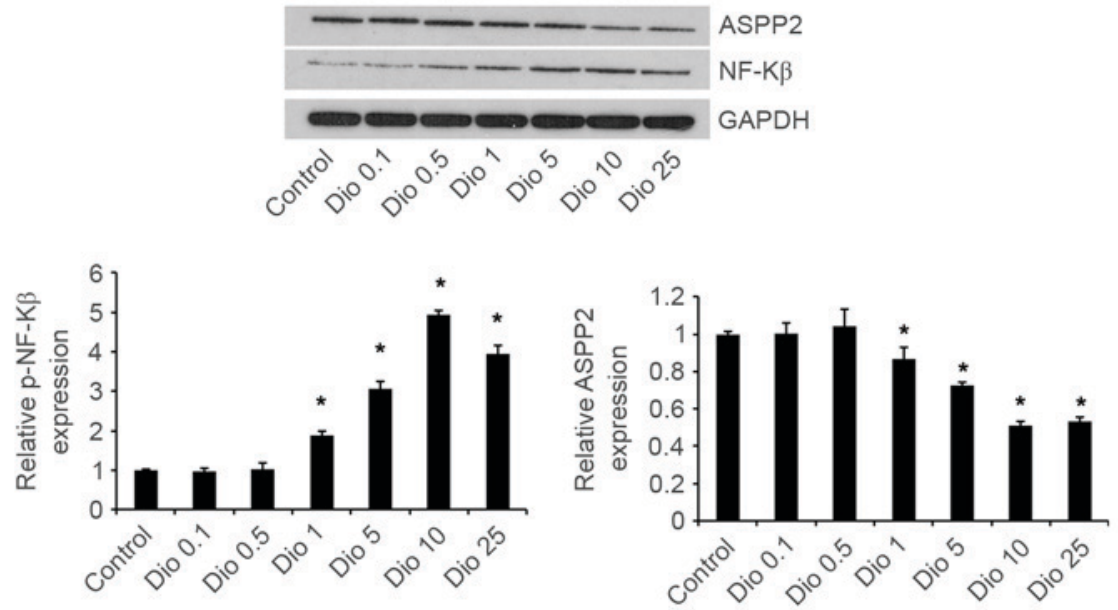

Figure 2. Effects of dioscin on the expression of ASPP2 and NF-K $\beta$ in MC3T3-E1 cells. MC3T3-E1 cells were treated with dioscin $(0.1,0.5,1,5,10$ and $25 \mu \mathrm{g} / \mathrm{ml}$ ) for $72 \mathrm{~h}$. Results were obtained from three independent experiments and are expressed as the mean \pm standard deviation. ${ }^{*} \mathrm{P}<0.01$, vs. control. Dio,

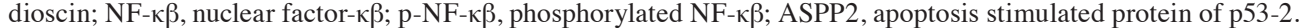

proliferation (Fig. 3B). These results indicated that dioscin induced osteoblastic cell proliferation and differentiation by increasing the expression of $\mathrm{p}-\mathrm{NF}-\kappa \beta$.

$N F-\kappa \beta$ is involved in dioscin-regulated autophagy of MC3T3-E1 cells. The present study subsequently examined whether ASPP2/NF- $\kappa \beta$ is involved in the dioscin-regulated autophagy of MC3T3-E1 cells. The MC3T3-E1 cells were treated with $1 \mu \mathrm{M}$ CAPE, an inhibitor of NF- $\kappa \beta$ activation, and with $10 \mu \mathrm{M}$ dioscin for $72 \mathrm{~h}$ to determine the effect of the ASPP2/NF- $\kappa \beta$ pathway on autophagy. As demonstrated in Fig. 3A, CAPE attenuated the dioscin-repressed expression of LC3B, and the immunofluorescence assay revealed that dioscin decreased autophagosome formation, which was reversed by
CAPE (Fig. 4). These results indicated that dioscin repressed autophagy via the NF- $\kappa \beta$ pathway in the MC3T3-E1 cells.

\section{Discussion}

Although dioscin has been demonstrated to have osteoprotective effects by inducing osteoblastic cell proliferation and differentiation (10), the role of dioscin in autophagy, and the mechanisms underlying the effects of dioscin on osteoblastic cell proliferation, differentiation and autophagy remain to be fully elucidated. Consistent with previous studies, the present study showed that the osteoblastic differentiation marker, ALP, was significantly upregulated by dioscin in a concentration-dependent manner. 
A

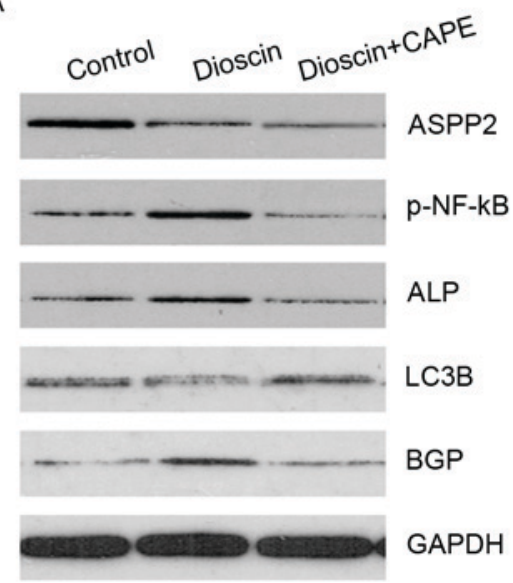

B

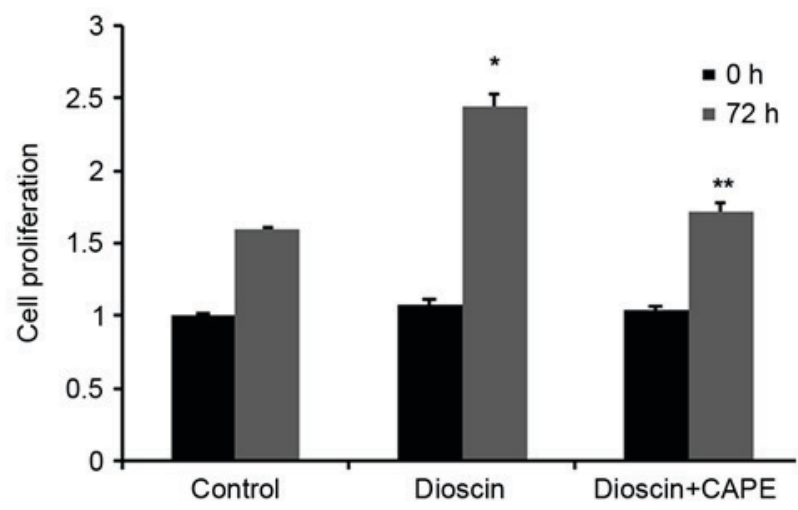

Figure 3. NF- $\kappa \beta$ is involved in dioscin-induced osteoblastic cell proliferation and differentiation. MC3T3-E1 cells were treated with $1 \mu \mathrm{M} \mathrm{CAPE}$ and $10 \mu \mathrm{M}$ dioscin for $72 \mathrm{~h}$. (A) Western blot analysis was used to detect the expression of p-NF- $\kappa \beta$, ASPP2, ALP, BGP and LC3B. (B) Cell proliferation was assessed using a 3-dimethylthiazol-2-y-(4,5)-2, 5-diphenyltetrazolium bromide assay. MC3T3-E1 cells were treated with $1 \mu \mathrm{M}$ CAPE and $10 \mu \mathrm{M}$ dioscin for $72 \mathrm{~h}$.

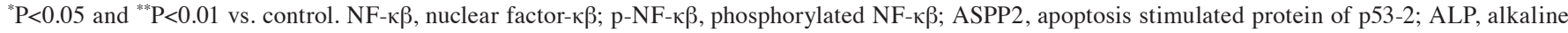
phosphatase; BGP, bone gla protein; LC3B, microtubule-associated protein 1 light chain 3 $\beta$; CAPE, caffeic acid phenethyl ester.

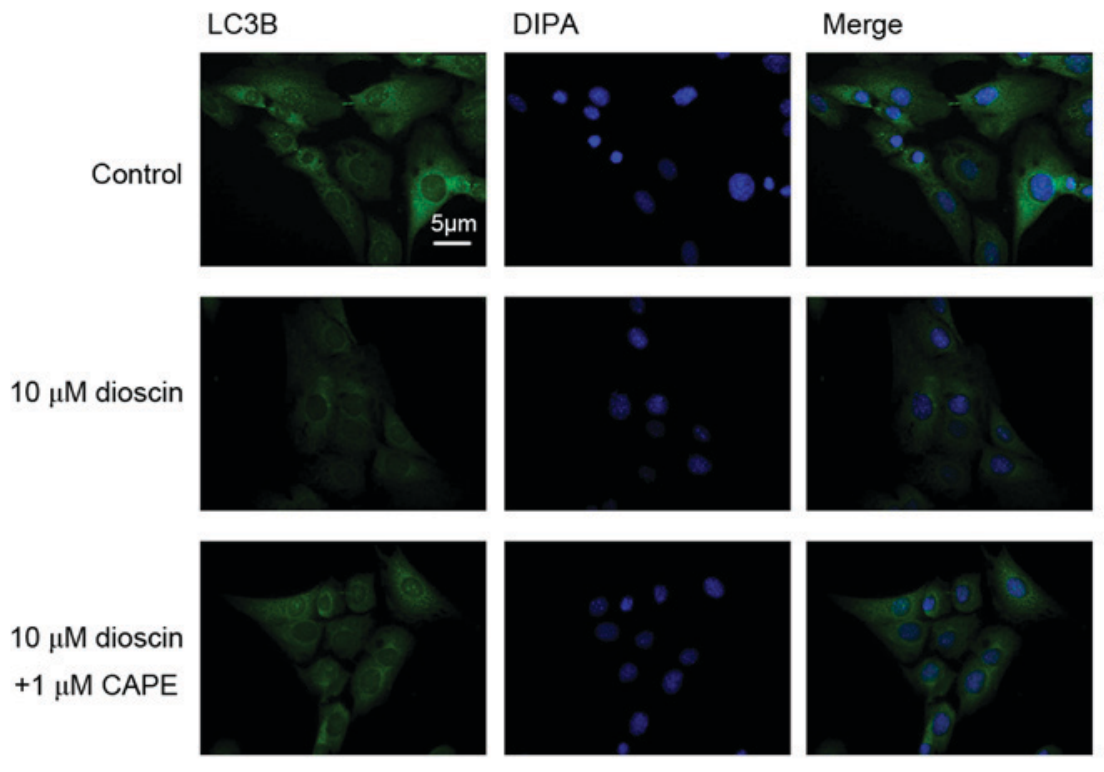

Figure 4. Dioscin regulates autophagy through the NF-к $\beta$ pathway. MC3T3-E1 cells were treated with $1 \mu \mathrm{M} \mathrm{CAPE}$ and $10 \mu \mathrm{M}$ dioscin for $72 \mathrm{~h}$. Autophagosome formation was observed under a fluorescence microscope. NF- $\kappa \beta$, nuclear factor- $\kappa \beta$; CAPE, caffeic acid phenethyl ester; LC3B, microtubule-associated protein 1 light chain 3 $\beta$; DIPA, diisopropanolamine dye.

Autophagy is a conserved homeostatic pathway by which intracellular components are delivered to lysosomes for degradation, and is critical for the removal of protein aggregates and damaged or excess organelles to maintain intracellular homeostasis (13). Autophagy is involved in a wide range of physiological and pathological processes, including cell survival, embryonic development and protection of the host from pathogen infection (18). The dysregulation of autophagy is known to be involved in various diseases, including tumorigenesis, disease of the immune system, heart disease and vascular disease (19-22). Previous studies have demonstrated that autophagy has a protective effect in the death of osteoblasts following exposure to lead chloride (23) and that impairment in autophagy aggravates the inhibitory effects of high glucose on osteoblast viability and function (24). However, the role of autophagy on dioscin-induced osteoblastic cell proliferation and differentiation remains to be fully elucidated. In the present study, it was identified that dioscin inhibited the expression of LC3B in a concentration-dependent manner in MC3T3-E1 cells, indicating that dioscin repressed autophagy in the MC3T3-E1 cells.

ASPP2 is a pro-apoptotic regulator of the ASPP family, which specifically stimulates the pro-apoptotic function of p53 (12). Previous studies have demonstrated that ASPP2 is involved in various biological pathways, including autophagy $(13,15,16)$. It has been reported that the NF- $\kappa \beta$ protein complex, which controls DNA transcription, cytokine production and cell survival, is directly regulated by ASPP2 (17). In the present study, it was identified that dioscin promoted the expression of ASPP2 and inhibited the 
expression of $\mathrm{NF}-\kappa \beta$ in the MC3T3-E1 cells, in a concentration-dependent manner. CAPE was then used to inhibit the activation of $N F-\kappa B$ and examine the effect of the ASPP $2 / N F-\kappa \beta$ pathway on osteoblastic cell differentiation, proliferation and autophagy. The results indicated that $\mathrm{NF}-\kappa \beta$ was involved in the dioscin-regulated differentiation, proliferation and autophagy of MC3T3-E1 cells.

In conclusion, the data obtained in the present study provide novel insights into the mechanisms by which dioscin induces differentiation and proliferation via the repression of autophagy. The ASPP $2 / \mathrm{NF}-\kappa \beta$ pathway was identified to be crucial in dioscin-induced osteoblastic cell differentiation and proliferation via the regulation of autophagy.

\section{Acknowledgements}

This study was supported by the National Natural Science Foundation of China (grant no. 81401815), the China Postdoctoral Science Foundation (grant no. 2015M582900) and the Jiangsu Postdoctoral Science Foundation (grant no. 1501146 C).

\section{References}

1. Takahashi A, Musy PY, Martins LM, Poirier GG, Moyer RW and Earnshaw WC: CrmA/SPI-2 inhibition of an endogenous ICE-related protease responsible for lamin A cleavage and apoptotic nuclear fragmentation. J Biol Chem 271: 32487-32490, 1996.

2. Aumsuwan P, Khan SI, Khan IA, Ali Z, Avula B, Walker LA, Shariat-Madar Z, Helferich WG, Katzenellenbogen BS and Dasmahapatra AK: The anticancer potential of steroidal saponin, dioscin, isolated from wild yam (Dioscorea villosa) root extract in invasive human breast cancer cell line MDA-MB-231 in vitro. Arch Biochem Biophys 591: 98-110, 2016.

3. Gao LL, Li FR, Jiao P, Yao ST, Sang H and Si YH: Apoptosis of human ovarian cancer cells induced by Paris chinensis dioscin via a $\mathrm{Ca}(2+)$-mediated mitochondrion pathway. Asian Pac J Cancer Prev 12: 1361-1366, 2011.

4. Lv L, Zheng L, Dong D, Xu L, Yin L, Xu Y, Qi Y, Han X and Peng J: Dioscin, a natural steroid saponin, induces apoptosis and DNA damage through reactive oxygen species: A potential new drug for treatment of glioblastoma multiforme. Food Chem Toxicol 59: 657-669, 2013.

5. Wei Y, Xu Y, Han X, Qi Y, Xu L, Xu Y, Yin L, Sun H, Liu K and Peng J: Anti-cancer effects of dioscin on three kinds of human lung cancer cell lines through inducing DNA damage and activating mitochondrial signal pathway. Food Chem Toxicol 59: 118-128, 2013.

6. Barile E, Capasso R, Izzo AA, Lanzotti V, Sajjadi SE and Zolfaghari B: Structure-activity relationships for saponins from Allium hirtifolium and Allium elburzense and their antispasmodic activity. Planta Med 71: 1010-1018, 2005.

7. LiH, Huang W, Wen Y, Gong G,Zhao Q and Yu G: Anti-thrombotic activity and chemical characterization of steroidal saponins from Dioscorea zingiberensis C.H. Wright. Fitoterapia 81: 1147-1156, 2010.

8. Lu B, Xu Y, Xu L, Cong X, Yin L, Li H and Peng J: Mechanism investigation of dioscin against $\mathrm{CCl}$-induced acute liver damage in mice. Environ Toxicol Pharmacol 34: 127-135, 2012.

9. Yu H, Zheng L, Xu L, Yin L, Lin Y, Li H, Liu K and Peng J: Potent effects of the total saponins from Dioscorea nipponica Makino against streptozotocin-induced type 2 diabetes mellitus in rats. Phytother Res 29: 228-240, 2015.
10. Zhang C, Peng J, Wu S, Jin Y, Xia F, Wang C, Liu K, Sun H and Liu M: Dioscin promotes osteoblastic proliferation and differentiation via Lrp5 and ER pathway in mouse and human osteoblast-like cell lines. J Biomed Sci 21: 30, 2014.

11. Ha SW, Weitzmann MN and Beck GR Jr: Bioactive silica nanoparticles promote osteoblast differentiation through stimulation of autophagy and direct association with LC3 and p62. ACS Nano 8: 5898-5910, 2014.

12. Shi Y, Han Y, Xie F, Wang A, Feng X, Li N, Guo H and Chen D: ASPP2 enhances oxaliplatin (L-OHP)-induced colorectal cancer cell apoptosis in a p53-independent manner by inhibiting cell autophagy. J Cell Mol Med 19: 535-543, 2015.

13. Song B, Bian Q, Zhang YJ, Shao CH, Li G, Liu AA, Jing W, Liu R, Zhou YQ, Jin G and Hu XG: Downregulation of ASPP2 in pancreatic cancer cells contributes to increased resistance to gemcitabine through autophagy activation. Mol Cancer 14: 177, 2015.

14. Liu K, Shi Y, Guo X, Wang S, Ouyang Y, Hao M, Liu D, Qiao L, Li N, Zheng J and Chen D: CHOP mediates ASPP2-induced autophagic apoptosis in hepatoma cells by releasing Beclin-1 from Bcl-2 and inducing nuclear translocation of Bcl-2. Cell Death Dis 5: e1323, 2014.

15. Wang Y, Wang XD, Lapi E, Sullivan A, Jia W,He YW, Ratnayaka I, Zhong S, Goldin RD, Goemans CG, et al: Autophagic activity dictates the cellular response to oncogenic RAS. Proc Natl Acad Sci USA 109: 13325-13330, 2012.

16. Xie F, Jia L, Lin M, Shi Y, Yin J, Liu Y, Chen D and Meng Q: ASPP2 attenuates triglycerides to protect against hepatocyte injury by reducing autophagy in a cell and mouse model of non-alcoholic fatty liver disease. J Cell Mol Med 19: 155-164, 2015.

17. Benyamini H, Leonov H, Rotem S, Katz C, Arkin IT and Friedler A: A model for the interaction between NF-kappa-B and ASPP2 suggests an I-kappa-B-like binding mechanism. Proteins 77: 602-611, 2009.

18. Hou H, Zhang L, Zhang L, Liu D, Mao Z, DU H and Tang P: Acute spinal cord injury in rats induces autophagy activation. Turk Neurosurg 24: 369-373, 2014.

19. Pan H, Chen L, Xu Y, Han W, Lou F, Fei W, Liu S, Jing Z and Sui X: Autophagy-associated immune responses and cancer immunotherapy. Oncotarget 7: 21235-21246,2016.

20. Nyhan MJ, O'Donovan TR, Boersma AW, Wiemer EA and McKenna SL: MiR-193b promotes autophagy and non-apoptotic cell death in oesophageal cancer cells. BMC Cancer 16: 101, 2016.

21. Schiattarella GG and Hill JA: Therapeutic targeting of autophagy in cardiovascular disease. J Mol Cell Cardiol 95: 86-93, 2016.

22. Orogo AM and Gustafsson $\AA \mathrm{B}$ : Therapeutic targeting of autophagy: Potential and concerns in treating cardiovascular disease. Circ Res 116: 489-503, 2015.

23. Lv XH, Zhao DH, Cai SZ, Luo SY, You T, Xu BL and Chen K: Autophagy plays a protective role in cell death of osteoblasts exposure to lead chloride. Toxicol Lett 239: 131-140, 2015.

24. Bartolomé A, López-Herradón A, Portal-Núñez S, García-Aguilar A, Esbrit P, Benito M and Guillén C: Autophagy impairment aggravates the inhibitory effects of high glucose on osteoblast viability and function. Biochem J 455: 329-337, 2013. 